\title{
Orbital emphysema following nose blowing as a sequel of a snowboard related head injury
}

\author{
Y Taguchi, Y Sakakibara, K Uchida, H Kishi
}

Br J Sports Med 2004;38:e28 (http://www.bjsportmed.com/cgi/content/full/38/5/e28)

A case of orbital emphysema as a sequel of a snowboard related head injury is reported. It is believed that a fracture of the medial orbital wall was caused by the increased intraorbital pressure when the patient hit his forehead on the snowy ground, allowing air to enter the orbit when he blew his nose. Wearing goggles may prevent this type of sports related injury.

E very kind of sport carried the potential risk of injury. Snowboarding is now one of the most popular winter sports. With the growth in the snowboarding population, snowboard related injuries have inevitably increased. More than half the snowboarders injured suffer closed head injuries, but most are mild. ${ }^{1}$ There have been few reports on sequels to mild head injury, ${ }^{2}$ though there are data on major head injuries requiring tertiary referral. Orbital emphysema is well documented but has not merited much attention, presumably because it is a benign self limiting condition. However, it assumes importance when complications occur such as infection or loss of vision because of pressure effects.

We report a case of orbital emphysema in a young man as a sequel of a mild snowboard related head injury and discuss the efficacy of wearing goggles to prevent this type of injury.

\section{CASE REPORT}

An otherwise healthy 19 year old man presented with swelling of his left eyelid. He fell while snowboarding and hit his left forehead on the snowy ground. He was not wearing a helmet or goggles. He denied any impact to the orbit or the nose. This blunt trauma did not result in any clinical problem and he continued snowboarding. Four hours later, he felt a "pop" in the left eye while blowing his nose. Immediately after this he became aware of narrowing of the palpebral fissure. He had no pain and no disturbance in his vision. He visited our outpatient clinic in the following day. Examination revealed no abnormality other than inflation of his left eyelid causing ptosis. Computed tomography with $2 \mathrm{~mm}$ slice thickness showed accumulation of air in the left orbit and an isolated medial orbital wall fracture, with significant displacement of the fractured fragments (figs 1 and 2). The patient was advised not to blow his nose forcefully. He improved with resolution of orbital emphysema over one week and subsequent follow up was unremarkable.

\section{DISCUSSION}

Orbital emphysema is a recognised but uncommon complication of diverse types of orbital and facial injury. Its appearance can be delayed until some time after the injury, at which point it is often precipitated by actions causing increased upper airways pressure, such as nose blowing or coughing. ${ }^{3}$ Direct blunt trauma to the orbit is a common cause of fracture of the lamina papyracea, presumably caused

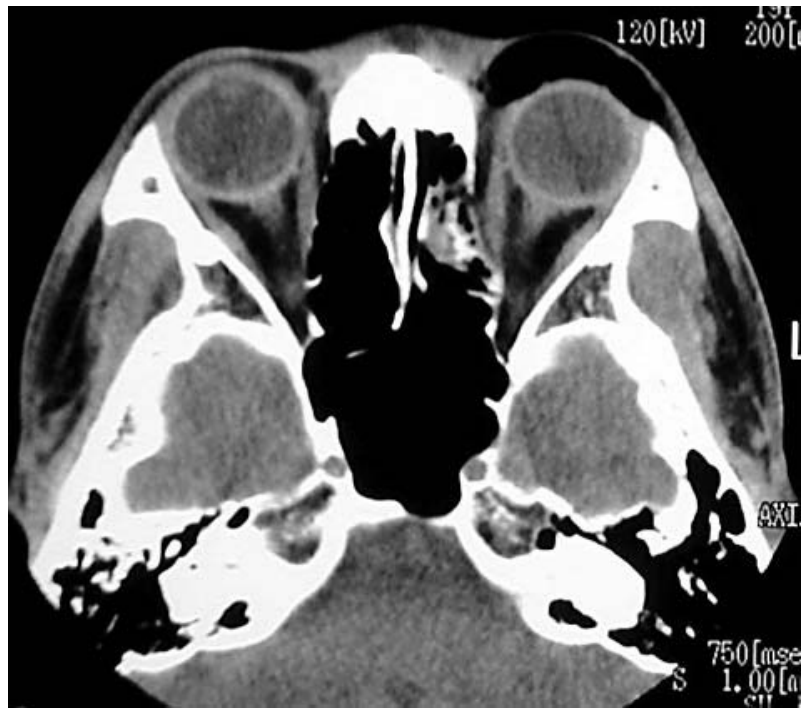

Figure 1 Computed tomography showing accumulation of air in the left orbit and a soff tissue protruded into the left ethmoid sinus. Note that the medial rectus muscle was not involved.

by the increased intraorbital hydraulic pressure or the buckling force, ${ }^{4}$ allowing communication between the orbit and the ethmoid sinuses. Very rarely orbital emphysema with indirect trauma $a^{5}$ or without any trauma ${ }^{67}$ has been reported.

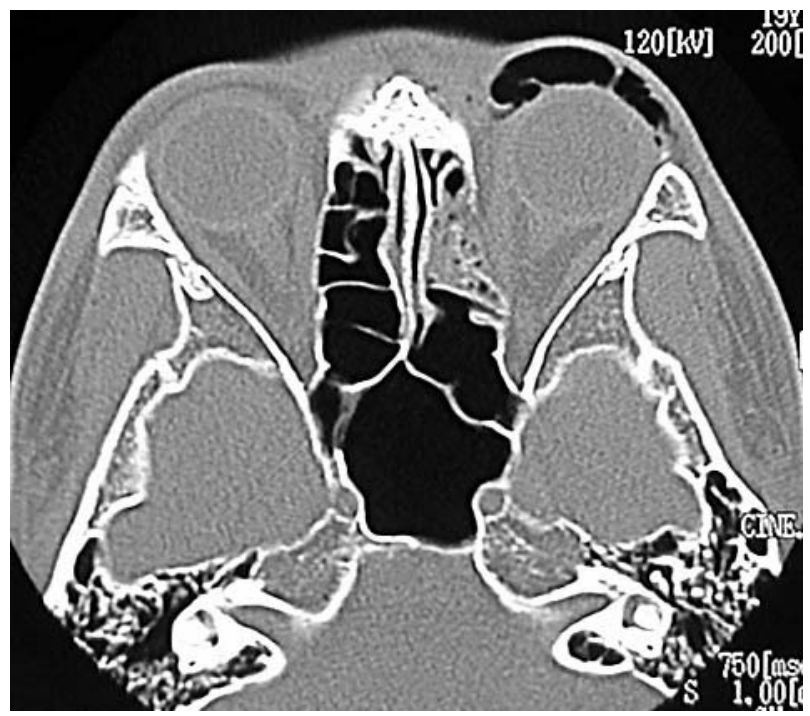

Figure 2 Computed tomography with bone window setting showing an isolated medial orbital wall fracture with significant displacement of the fractured fragments. 


\section{Take home message}

- More than half the injuries suffered by snowboarders are mild head injuries, but there have been few reports on these. No role for goggles other than to prevent eye injury appears to have been recognised.

- A blow out fracture of the orbit is described, a type of snowboard related injury not reported previously. Wearing goggles may prevent such fractures occurring during snowboarding.

Although direct orbital or nasal injury was denied in our case, the abrupt increase in intraorbital pressure appeared to be the cause of the fracture in the medial orbital wall because medial displacement of the fractured fragments suggested the usual type of blow out fracture. We believe that accumulated snow provided a considerable force to the orbit when the patient hit his forehead on the snowy ground, because he was not wearing goggles. Hence, wearing goggles may have prevent the fracture of the medial orbital wall in our case.

With the progress in computed tomography technology, the medial orbital wall, which is known theoretically to be the weakest point of the orbital wall, has been reported to be the most common site of pure orbital blow out fracture. ${ }^{4}$

Orbital emphysema is a benign self limiting condition. As emphysema usually resolves over a period of about two weeks, ${ }^{3}$ no treatment is required unless there are associated visual symptoms. However, it should be realised that compressive or tension orbital emphysema can cause severe visual loss. ${ }^{89}$ As fibrosis around the fracture sites of the lamina papyracea is expected to occur within two weeks, possibly closing a sinus-orbital communication, ${ }^{10}$ all patients with orbital emphysema should avoid nose blowing, sneezing, coughing, vomiting, or the Valsalva manoeuvre for at least two weeks after the injury. This is probably a good recommendation for all patients who might have medial orbital fractures, because small fractures may not always be readily seen on imaging studies. ${ }^{5}$
As participation in snowboarding rapidly increases, considerable numbers of snowboarders may suffer mild head injuries. Physicians involved with snowboard related head injuries should be aware that a trivial head injury may cause fractures of the lamina papyracea resulting in orbital emphysema following nose blowing. To prevent this sequel, it is recommended that all snowboarders wear goggles.

\section{Authors' affiliations}

Y Taguchi, Y Sakakibara, K Uchida, Division of Neurosurgery, St Marianna University, Yokohama City Seibu Hospital, Yokohama, Kanagawa, Japan

H Kishi, Division of Neurosurgery, Asao General Hospital, Kawasaki, Kanagawa, Japan

Correspondence to: Dr Yoshio Taguchi, Division of Neurosurgery, St Marianna University Yokohama City Seibu Hospital, $1197-1$ Yasashicho, Asahi-ku, Yokohama, Kanagawa 241-0811, Japan; ytaguchi@ marianna-u.ac.jp

7 September 2003

\section{REFERENCES}

1 Nakagichi H, Fujimaki T, Ueki K, et al. Snowboard head injury: prospective study in Chino, Nagano, for two seasons from 1995 to 1997. J Trauma 1999:46:1066-9.

2 Uzura M, Taguchi Y, Matsuzawa M, et al. Chronic subdural haematoma after snowboard head injury. Br J Sports Med 2003;37:82-3.

3 Kaplan K, Winchell GD. Orbital emphysema from nose blowing (letter). N Engl J Med 1968;278:1234.

4 Burm JS, Chung CH, Oh SJ. Pure orbital blowout fracture: new concepts and importance of medial orbital blowout fracture. Plast Reconstr Surg 1999; 103:1839-49.

5 Brown SM, Lissner G. Orbital emphysema following remote skull trauma. Ophthal Plast Reconstr Surg 1995;11:142-4.

6 Mohan B, Singh KP. Bilateral subcutaneous emphysema of the orbits following nose blowing. J Laryngol Otol 2001;115:319-20.

7 Monaghan AW, Millar BG. Orbital emphysema during air travel: a case report. J Cranio Maxill Surg 2002;30:367-8.

8 Wood BJ, Mirvis SE, Shanmuganathan K. Tension pneumocephalus and tension orbital emphysema following blind trauma. Ann Emerg Med 1996;28:446-9.

9 Zachariades N, Mezitis M. Emphysema and similar situations in and around the maxillo-facial region. Rev Stomatol Chir Maxillofas 1988;89:375-9.

10 Seiff SR. Atmospheric pressure changes and the orbit: recommendations for patients after orbital trauma or surgery. Ophthal Plast Reconstr Surg 2002;18:239-41. 